\title{
Control system for electrohydraulic drive of a mobile sinking hoisting plant
}

\author{
Anton Kurochkin ${ }^{1, *}$,Vladimir Vagin ${ }^{1}$, Anton Karpesh ${ }^{1}$, and Natalja Dyorina ${ }^{1}$ \\ ${ }^{1}$ Nosov Magnitogorsk State Technical University, Magnitogorsk, Russia
}

\begin{abstract}
The article presents the results of the control system development for the electro hydraulic drive of a mobile sinking hoisting plant. Increasing the technical performance of mobile sinking hoisting plants is possible by increasing their productivity, reducing their massiveness and increasing the durability of tractional organs and a number of units and parts of lifting machines. However, the increase in productivity, in its turn, causes the need to increase the suspended load on the traction organs, which causes a significant increase of the dynamic loads in the elastic elements in the transient operation modes of the hoisting plant. One of the ways to reduce constantly acting dynamic loads of vibration character is the use of a gearless hydraulic drive with damping properties. But, nevertheless, the hydraulic drive possibilities are limited for large vibration amplitudes of dynamic forces. The damping properties of the hydraulic drive can be increased by using control system, which allows realizing effective control of the electro hydraulic drive of the hoisting plant.
\end{abstract}

\section{Introduction}

Electro-hydraulic motors of mobile sinking hoisting plants operate under conditions that differ significantly from the operating conditions of stationary lifting motors. These differences consist in the fact that hydroelectric drives are used in mobile sinking hoisting plants with continuously variable lifting heights, with different lifting capacities at different lifting speeds [1].

Variable suspended loads - from the maximum one, equal to the weight of the lifted load and the lifting vessel, to a minimum one with weight equal only to the trailing device, require the stable lifting motor operation at different driving moments. The necessity of frequent descent of loads requires from the hydraulic motor of the sinking hoisting machine to work continuously for a negative value of the driving moments, i.e., in the braking mode $[2,3]$.

In connection with these features, it becomes necessary to consider possible ways of controlling the electric drive of a sinking hoist.

\footnotetext{
* Corresponding author: antoshey@mail.ru
} 


\section{Methodology}

\subsection{Start-up and even movement when lifting the load}

The necessary magnitude of the motor torque when lifting the load and people is set automatically in accordance with the load [4].

Before starting the lifting hydraulic motor, the lift control handle is set to zero and the cylinder block of the controlled pump is in neutral position at which the pump feed is zero $[5,6]$. During the start-up period, the operator of the sinking hoisting plant re-moves the control handle in one direction or the other (depending on the bar-ban rotation direction) and gradually withdraws the cylinder block of the regulated pump from the neutral position, thus ensuring a smooth supply of hydraulic fluid to the lifting hydraulic motor.

The mechanical characteristics of the high-torque electro hydraulic drive of the sinking lifting are shown in Fig.1.

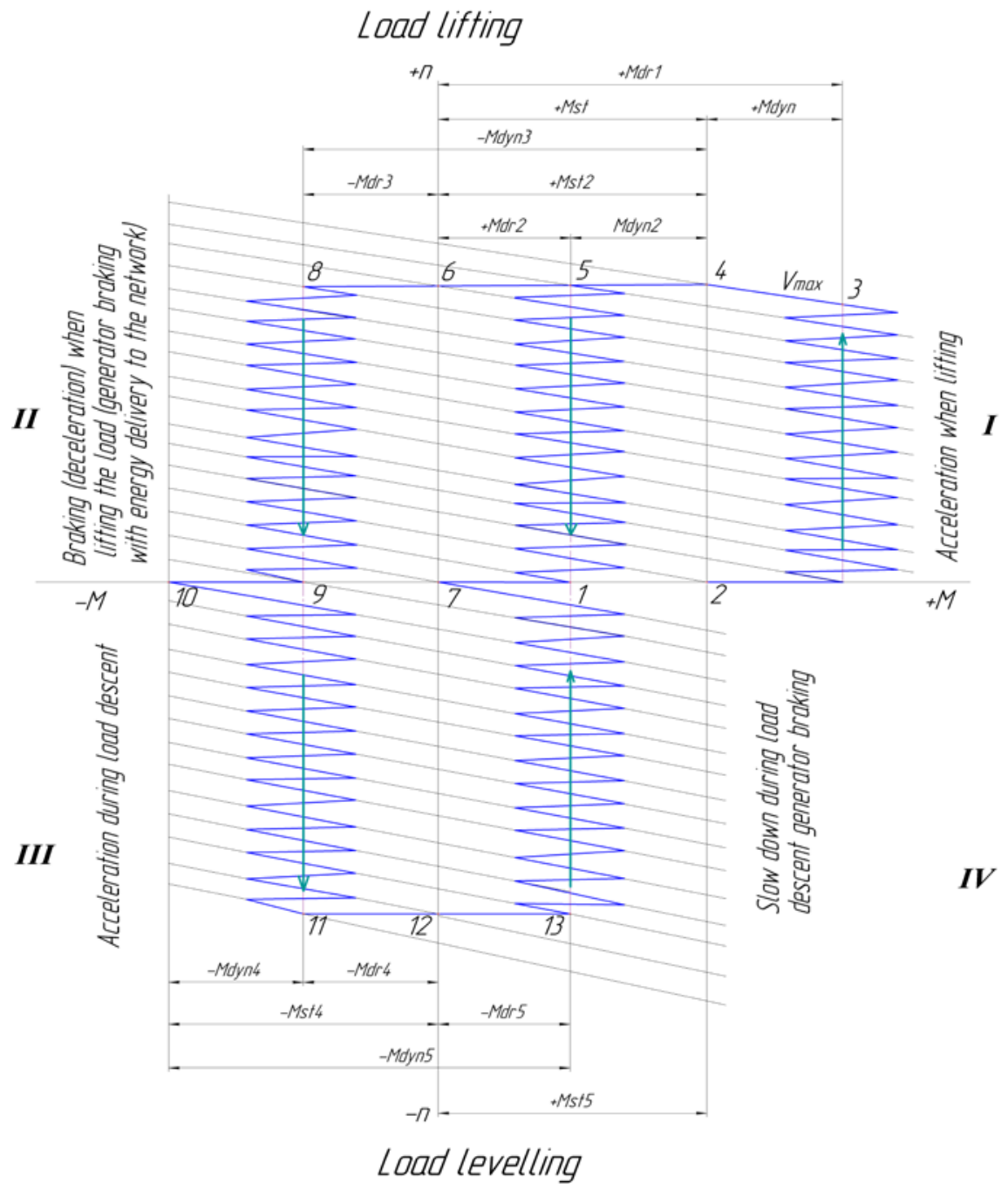

Fig. 1. Control methods, operating modes and mechanical characteristics of high-torque electro hydraulic drive of mobile sinking hoisting plants. 
Start of the lifting hydraulic motor is carried out with the help of the handle of the hoisting machine or the monitoring electrohydromechanical control system [7]. The cylinder block of the regulated pump is moved from the neutral position and the pump supplies the working fluid to the lifting hydraulic motor. After the pump is turned on, the hydraulic motor develops the torque necessary for tensioning the lift system, pointing all the gaps and compensating for leaks in the hydraulic motor under normal load lifting conditions, and revising the traction organ when operating at low speeds equal to $0,3 \mathrm{~m} / \mathrm{s}$ and the value of $\mathrm{M} d r \approx 0.4 \mathrm{Mrat}[8]$.

When the handle of the lifting machine is moved smoothly, the hydraulic motor generates the torque reaching at point 2 the values of the static torque Mst. After this, the acceleration starts, i.e., the movement of the lifting system when changing from one mechanical characteristic to another from point 2 to point 3 .

The described start-up process occurs at the maximum lifted load on mechanical characteristics, each of which corresponds to a certain pump feed and pressure proportional to the load on the hydraulic motor shaft. The increase in speed corresponds to a constant average acceleration of uniformly accelerated movement of the lifting vessel in the trunk. The average value of the driving torque remains almost constant during the start-up period of $\mathrm{M} d r 1$.

By means of the monitoring control system acting on the supply unit of the controlled pump, we can change the supply of hydraulic fluid to the hydraulic motor. Thus, any law of acceleration change can be obtained [9].

At point 3, the hydraulic motor has the characteristic with a constant pump feed, where at point 4 its moment becomes equal to static, and the load lifting speed is equal to the maximum calculated one for a given depth of the trunk. Thus, there is a constant speed period of the lifting machine.

\subsection{Slowing down and stopping when lifting the calculated cargo}

The slowdown of the hydraulic motor, and, consequently, of the hoisting machine can be carried out in various ways, depending on the magnitude of the driving moments. Slowdown in the driving mode can be realized with a change in speed from point 5 to 1 , in free coasting - from point 6 to 7 or in the mode of generator braking from point 8 to point 9 .

At points 1 and 9, the sensor installed at the place of the time -loading of the hoisting lifting vessel, tearing the control circuit, and the machine stops and stops short with a mechanical brake.

This corresponds to the transition from point 1 to point 7 or when applying a brake from point 9 to 7 .

This is how start-up, acceleration, steady-state motion, deceleration and breaking of the hoisting plant system take place. The characteristics of the hydraulic drive are shown in the first and second quadrants in Fig.1.

\subsection{Acceleration, deceleration and braking the hydraulic drive during descent of the load}

The mode of descent of cargoes is carried out in the following sequence. The hoist is disinfected by a mechanical brake. Under the action of the gravity of the lowered load and the activation of the hydraulic drive for descent, the acceleration from the point 7 to the point 10 and then the point 11 during the transition from one mechanical characteristic of the hydraulic drive to the other is accelerated. At point 12, the load is launched at a constant speed. 
When the control handle is moved reversely (in Figure 1 from point 12 to point 13), the load will be retarded from point 13 to point 7 . The braking of the hoisting plant will occur with a hydraulic motor. In this case, the hydraulic motor will operate in the pump mode, the pump in the hydraulic motor mode, and will untwist the induction motor of the pump to a super synchronous speed, i.e. in the generator mode giving electric energy to the network. The mode of cargo descent is shown in III and IV quadrant.

To reduce the dynamic component and to damp the load of hoisting in the braking modes, the studies of the new traction organs use for lifting machines were carried out [10, $11]$.

Realization of the qualitative control functions of the electrohydraulic drive of the hoist is possible by use of various types of regulators.

\section{Conclusion}

The use of an electrohydraulic drive for mobile sinking hoisting plants provides any steady speed in the modes of acceleration, deceleration and stopping of the hoisting machine, provides the improved control quality. Control system allows to reduce the working time and avoid overshoot.

Brake modes of the hoisting plant are provided in the generator operating mode of the electro-hydraulic drive.

Generator braking provides stiff and stable performance over a wide range of speeds without overloading the hydraulic drive and provides the necessary control accuracy, as well as significantly reduces the electrical energy consumption of the sinking hoist.

The use of an electrohydraulic drive ensures that the hoisting machine stops without using a mechanical brake, which can serve mainly to stopper the machine and as an emergency one.

\section{References}

1. V.S. Vagin, A.I. Kurochkin, A.A. Karpesh, Proc. Eng. 206, 21(2017)

2. A.I. Kurochkin, A.D. Kol'ga, V.S. Vagin, A.M. Filatov, Extr. act. treat. \& appl. of nat. st. 126 (2016)

3. A.I. Kurochkin, A.D. Kol'ga, V.S. Vagin, Extr. act. treat. \& appl. of nat. st. 130 (2016)

4. A.A. Karpesh, V.S. Vagin, A.I. Kurochkin, K.A. Melkomukov, Act. Prob. of mod. Sci., tech. \& ed. 123 (2017)

5. V.S. Vagin, A.M. Filatov, A.I. Kurochkin, Act. Prob. of mod. Sci., tech. \& ed. 170 (2012)

6. L.I. Kantovich, V.S. Vagin, A.I. Kurochkin, Min. inf. \& anal. bull. 1396 (2016)

7. V.S. Vagin, A.M. Filatov, A.I. Kurochkin, Extr. act. treat. \& appl. of nat. st. 65 (2013)

8. A.A. Karpesh, V.S. Vagin, A.I. Kurochkin, Act. prob. of incr. effic. 155 (2016)

9. V.S. Vagin, A.M. Filatov, A.I. Kurochkin, Min. inf. \& anal. bull. 6254 (2014)

10. N.G. Kartavyy, V.V. Zotov, Min. J. 175 (2009)

11. V.V. Zotov, Min. inf. \& anal. bull. 10287 (2006) 